\title{
Urgences
}

\section{Les hôtes du rez-de-chaussée}

\section{Gilles Lamontagne et Martine Lévesque}

Numéro 27, mars 1990

Images imaginaires

URI : https://id.erudit.org/iderudit/025580ar

DOI : https://doi.org/10.7202/025580ar

Aller au sommaire du numéro

Éditeur(s)

Urgences

ISSN

0226-9554 (imprimé)

1927-3924 (numérique)

Découvrir la revue

Citer cet article

Lamontagne, G. \& Lévesque, M. (1990). Les hôtes du rez-de-chaussée. Urgences, (27), 71-87. https://doi.org/10.7202/025580ar d'utilisation que vous pouvez consulter en ligne.

https://apropos.erudit.org/fr/usagers/politique-dutilisation/ 


\title{
Les hôtes du rez-de-chaussée *
}

\section{Gilles Lamontagne (avec la collaboration de Martine Lévesque)}

\author{
3
}

En mars 1880, Le journal des débats inaugure en France la tradition du feuilleton en confiant à Julien-Louis Geoffroy sa chronique dramatique que les lecteurs pourront désormais lire au «rez-de-chaussée». Par la suite, le feuilleton s'ouvre peu à peu à la critique littéraire, musicale, artistique et scientifique de sorte que bientôt chaque jour de la semaine se voit assigner une matière différente.

Puis, timidement, le "rez-de-chaussée» finit par accueillir la fiction. Par exemple, le journal Le siecle publie les 5, 27 août et 10 octobre 1836 des chapitres du roman espagnol Lazarille de Tormes (H. de Mendoza) traduits et présentés par Louis Viardot. Quelques semaines plus tard, La presse fait paraître de son côté le premier roman français écrit pour le feuilleton: La vieille fille de Balzac. Le succès fut immédiat et suscita aussitôt une concurrence effrénée entre les journaux et une émulation fiévreuse parmi les écrivains: Lamartine publie Le tailleur de pierres de Saint-Point, Alexandre Dumas Le capitaine Paul, Eugène Sue Le journal d'un inconnu, Frédéric Soulié Les mémoires du diable, etc.

On mesure le chemin parcouru: le feuilletoniste, de journaliste qu'il était exclusivement, s'est transformé en romancier, auteur de ce qu'on a commencé à appeler le «romanfeuilleton", qu'il ne faut pas confondre avec le "feuilletonroman " 1 . La différence entre les deux est en effet importante:

* Le document qui suit est composé d'extraits du rapport de recherche (CRSH) intitulé Les feuilletons litteraires parus dans les joumaux du Bas-Saint-Laurent de 1861 à 1950 (UQAR, 1987, 204 p.). Les extraits ont été découpés dans le premier chapitre, ainsi intitule (parce que le rez-de-chaussée, comme on disait au XIX siècle, désigne la partie inférieure d'une page de journal réservée à une rubrique particulière), dans l'annexe l et dans la note introduisant l'annexe II.

1 En pratique, pourtant, cette confusion existe. Alors que Pierre Noriey, par exemple, se réfère à La vieille fille de Balzac pour preciser : " le feuilleton-roman, comme on disait à l'époque, est lancé [...] et cet enfant incestueux du journalisme et de la littérature est né " ( Le roman-feuilleton ", Le crapouillot, mars 1934, p. 34), Patrice Caillot écrit dans Les mystères du rez-de-chaussée, Paris, Bibliothèque nationale, 1987, p. 5: «Ainsi, peu à peu, remarque JeanLouis Bory, à côté du feuilleton-roman, "consacré à la publication de n'importe 
alors que le feuilleton-roman désigne le découpage en tranches, pour publication par un journal, d'un roman déjà édité, le roman-feuilleton est une fiction conçue pour un journal particulier et son lecteur type. C'est en quelque sorte un roman écrit sur mesure. En ce sens, comme le note Roger Bellet, le journal "crée» le roman, lui impose ses lois, le rend "populaire» et "quotidien" comme lui ${ }^{2}$. D'où la fameuse mécanique d'enchaînement et de rebondissement mise au point par les feuilletonistes que la revue Paris-journaliste, en 1854, appelait plaisamment les « romanciers à longue queue ».

Le journal Satan (29 janvier 1868), avec un humour prédadaïste, donne la recette pour écrore un roman-feuilleton à succès :

Mettez dans votre cornue 25 duels, 12 empoisonnements, 1 enfant perdu, 1 agent de police, 2 forçats, 4 mouchards, 1 beau jeune homme mystérieux, 3 assassinats et 2 suicidés; faites chauffer à blanc et étendez sur le papier avec une plume d'oie, coupez en tranches que vous servirez une à une, en renvoyant chaque fois "au prochain numéro" et ouvrez avec confiance votre coffre-fort. ${ }^{3}$

C'est presque à la lettre ce que fit le plus flamboyant des feuilletonistes français, Ponson du Terrail, le célèbre père de Rocambole 4 . "Le jour, écrit-il en 1859 , où j'ai lu cette phrase: "Quelle était cette main? Quelle était cette tête? Suite au

quel roman ", s'impose le roman-feuilleton, "consacré à la publication d'un roman fabriqué pour le feuilleton en accord avec le gout supposé du plus grand nombre"." Jean Tortel note dans le même sens : "l'expression "feuilletonroman " employée vers 1840 pour désigner n'importe quel texte romanesque publié en rez-de-chaussée de journal " (Europe, $n^{\circ} 542$, juin 1974, p. 159). Avec le temps, roman-feuilleton et feuilleton finiront par désigner tout roman publié en tranches dans un journal, peu importe la manière dont il aura été conçu.

2 Roger Bellet, "Le feuilleton", dans Presse et journalisme sous le second empire, coll. "Kiosque ", Paris, Colin, 1967.

3 lbid.

4 Ses ennemis, jaloux de ses succes, l'appelaient Tronçon du Poitrail ! Quant à Rocambole, il s'agit d'un a personnage qui joue un rôle considérable dans l'ceuvre féconde de Ponson du Terrail (notamment dans Les drames de Paris qui groupent une trentaine d'ouvrages, 1859-1884). Héros pittoresque qui connaît des aventures compliquées et extravagantes (d'oú l'adjectif " rocambolesque ") dans le Paris du Second Empire, Rocambole est un maittre mystérieux [qui] asservit les hommes aussi bien que les femmes, mais que sa nature [...] porte toujours à [se] ranger du cóté du faible contre le fort. II est à l'origine de plusieurs héros du roman-feuilleton (Fantomas, etc.)" (Le petit Robert 2 : dictionnaire universel des noms propres). 
prochain numéro", j'ai compris que ma voie était trouvée. » 5 A dix-huit ans, il emboite donc le pas aux feuilletonistes qui avaient alors toute son admiration: Frédéric Soulié, Eugène Sue et Dumas père. À vingt-quatre ans (1853), il était célèbre. Ayant pris soin de s'inventer une généalogie qui le reliait à nul autre que Bayard, il poussa l'audace jusqu'à s'affubler du titre de vicomte. Travailleur acharné, il en arriva à pouvoir réaliser de véritables prouesses comme celle de mener de front, sur cinq tables, la rédaction de cinq feuilletons différents pour autant de journaux concurrents! Des poupées représentaient les personnages vivants des romans qu'il était en train d'écrire. Chaque fois que, pour les fins de l'intrigue, il devait en faire mourir un, la poupée correspondante prenait le chemin d'un placard qu'il avait baptisé le «cimetière». Lorsqu'il lui arrivait, par inadvertance, d'expédier trop tôt dans l'autre monde tel ou tel personnage, Ponson du Terrail ne se laissait pas décontenancer pour autant: il intitulait le feuilleton du lendemain "Celui que l'on croyait mort" ! La fébrilité avec laquelle il écrivait explique les nombreuses perles que l'on s'est amusé à relever dans ses romans. La plus célèbre, passée en proverbe, demeure sans doute celle-ci : «La main de cet homme était froide comme celle d'un serpent " 6 !

Alexandre Dumas fut à peine moins prolifique. Il lui arriva aussi de faire paraître quotidiennement et simultanément quatre romans dans quatre journaux différents. Mais, contrairement à Ponson qui travaillait en solitaire, Dumas avait des "nègres" parmi lesquels on retrouve nul autre que Gérard de Nerval 7. Ainsi que la plupart des feuilletonistes, Dumas était payé à la ligne comme l'atteste un contrat qu'il signe avec le journal Le constitutionnel (en 1845?). Aux termes dudit contrat, il s'engageait à fournir au journal 100000

5 Roger Bellet, op. cit., p. 208.

6 Les mystères du rez-de-chaussée, p. 1. À la fin de son article, « Le romanfeuilleton ", Pierre Noriey en donne une collection; en voici quelques-unes : "Son chapeau bosselé, déchiré, n'avait plus figure humaine" (Rocambole); « II avait ce merveilleux accent moitie espagnol, moitie anglais, qui accuse l'origine brésilienne " (ibid.); "O'Penny est un homme sauvage des terres australes dont je vais vous dire l'histoire sur l'air et avec les instruments du pays... Alors la Bohémienne prend un tambour de Basque... et chante un air... indien " (ibid.)!

7 Pierre Noriey, ibid., p. 35. Dans son article "La littérature populaire française au dix-neuvième siècle" (Revue canadienne de litterature comparée, vol. IX, $n^{\circ} 3$, septembre 1982), Marc Angenot rappelle qu'un feuilletoniste moins en vue, Pierre Decourcelle, eut Saint-Pol Roux comme " nère ". 
lignes annuellement à 1 franc 50 la ligne (il ne versait que 20 centimes à ses "nègres"). Dans ces conditions, "il faudrait être un saint pour n'être pas tenté de tirer à la ligne» pour reprendre l'expression de Pierre Noriey. Aussi, Dumas s'empressa-t-il d'inventer un personnage taciturne, le fameux valet Grimaud, qui « a le génie du monosyllabe»:

- Eh! bien?

- Rien.

- Rien!

- Rien.

- Comment?

- Rien, vous-dis-je.

- C'est impossible.

- Puisque je vous le dis.

- En es-tu bien sûr?

- Certainement.

- C'est un peu fort.

- C'est comme cela! 8

Douze lignes, dix-huit francs! Il n'est pas étonnant qu'avec de tels procédés, Dumas ait gagné 180000 francs pour la seule année 1846 !

Quand donc Sainte-Beuve, en septembre 1839, analysait dans $L a$ revue des deux mondes le phénomèene nouveau de "la littérature industrielle», en parlant du feuilleton, il n'avait encore rien vu. Pour nous en tenir à Ponson du Terrail, qu'il suffise de rappeler que les aventures de Rocambole, toutes publiées en feuilletons et inventées sans l'aide du moindre collaborateur, couvrent une trentaine de volumes!

Le journal de Fraserville a publié du 27 mai 1892 au 23 juin 1893 , sous l'anonymat, le premier des quatre volumes qui composent Les voleurs du grand monde de Ponson du Terrail, édité chez E. Dentu en 1869-1870; en tout, 1364 p. L'héroïne, Olympe Mignot, "était une intelligence supérieure, et comme on le verra plus tard, d'autant plus pessimiste 
qu'elle était portée au mal "( 8 juillet 1892). Ambitieuse, calculatrice, elle a appris de son voisin Loudéac que le vieux marquis de Faustinières, connu de tous sous le nom de Cabestan, possède une immense fortune. À partir de ce moment, Olympe n'a qu'un seul but: mettre la main sur l'argent de Cabestan. Sur les conseils de Loudéac, vieux pilote qui a longtemps sillonné les mers avec le marquis, elle épouse secrètement le fils adoptif de ce dernier, Cartahut.

Il y a plus de cinquante ans, Cabestan avait épousé, aux Indes, une jeune princesse dont il avait eu un fils. Tout le monde ignore ce fait. Devenu vieux, Cabestan charge Cartahut de retrouver le jeune "prince» pour en faire son héritier.

Quand Cartahut confie à Olympe le secret de sa mission, celle-ci entre dans une rage folle: "Ah ! l'imbécile! dit-elle, ah! le misérable! les millions de Cabestan ne sont pas pour lui et je suis sa femme. Ah! ah! ah! mais c'est son arrêt de mort qu'il a lui-même prononcé " (18 novembre 1892).

Entre-temps, un neveu de Cabestan, le baron Victor, s'est épris d'Olympe qui se sert de lui pour se débarrasser de son mari, Cartahut. Ce dernier mort, Olympe trouve dans ses poches un papier qu'il avait été chargé par Cabestan de remettre à son fils pour lui permettre d'hériter de sa fortune. Désormais elle-même en possession du fameux papier, Olympe croit qu'elle pourra mettre la main sur l'héritage. Ce qu'elle ne sait pas, c'est que quelques heures avant d'expirer, Cabestan, pressentant que Cartahut est mort, avait pris de nouveaux arrangements: il avait confié à son domestique Mériadec un autre papier, semblable au précédent. De sorte que désormais, tout(e) prétendant(e) à l'héritage devra produire les deux papiers, sauf s'il s'agit du "prince" ou de l'un ou l'autre de ses descendants; dans ce cas, un seul papier suffira...

Ainsi, Olympe se leurre. Après la mort de son mari, elle a épousé un autre neveu de Cabestan, M. de Gonidec, qui avait d'abord refusé d'en faire sa femme sous préterxte qu'elle n'était pas de son rang. Olympe lui avait par la suite confié: "Pauvre, de naissance obscure, j'ai toujours rêvé la fortune et un rang dans le monde. Vous aviez brisé mon cœur, j'en jetai les morceaux sanglants par le fenêtre " (27 janvier 1893)! Et Ponson du Terrail d'enchaîner: "La jeune fille n'existait plus. elle avait fait place pour toujours à la femme ambitieuse qui ne reculerait devant aucun moyen pour satisfaire son ambition" (ibid.). 
Son ambition, Olympe va la promener dans les trois autres volumes que Le journal de Fraserville n'a pas publiés. Au rythme où le premier a paru, il lui aurait fallu trois années supplémentaires! Mais nous en savons encore assez pour nous familiariser avec le genre.

Le héros, le plus souvent masculin, cède ici la place à une femme. Et quelle femme! Par opposition à la féminité mystérieuse, l'un des topiques de la littérature feuilletonesque, elle incarne la féminité tragique, topique du mélodrame dont le premier feuilleton de Ponson du Terrail, La nonne sanglante, est un autre bel exemple. Le catalogue des romans populaires du XIX siècle contient d'autres titres qui laissent eux aussi deviner, derrière la femme, la sorcière: La sirène de l'enfer, La belle dangereuse, Sarah la mangeuse d'hommes, Une haine de femme ${ }^{9}$.

Sans statut social, donc sans argent, Olympe ambitionne d'acquérir à la fois un nom et la fortune par tous les moyens y compris le mensonge, la tromperie, le meurtre... On la voit donc se démener dans l'univers manichéen si caractéristique du conte et du roman populaire: d'un côté les bons, qui seront ses opposants puisqu'elle est un être maléfique, et de l'autre les méchants qui lui serviront d'adjuvants.

Parmi les méchants, au premier chef, Loudéac, l'ancien pilote qui a İongtemps navigué avec Cabestan. Il est un être perfide, dévoré lui aussi par l'ambition. Il se dit l'ami de Cabestan et de Cartahut, mais c'est pour mieux les tromper. Quant au jeune baron Victor, il est plus faible que méchant. Rien n'empêche qu'aveuglé par son amour pour Olympe, il se laisse persuader par elle d'éliminer son mari Cartahut au cours d'un combat dans lequel il trouvera la mort. Gonidec, enfin. Après avoir épousé Olympe, il devient son complice pour mettre la main sur l'héritage tant convoité de son oncle.

Dans Les voleurs du grand monde, les bons sont incarnés d'abord par Cartahut, l'orphelin que Cabestan a adopté. (Notons, en passant, la présence de l'orphelin, si fréquente dans les romans populaires.) C'est un être d'une parfaite loyauté, honnête, tout à fait désintéressé. Il ne souhaite rien d'autre que l'amitié et l'affection de son père adoptif auquel il voue une reconnaissance indéfectible. Mériadec, le domestique, est 
lui aussi un être bon, tout entier dévoué aux intérêts de son maître Cabestan. Il constitue un adversaire redoutable et acharné pour Olympe et Gonidec. Ayant acquis la certitude que le fils de Cabestan est mort, il finit par retrouver la trace de sa femme et de ses enfants... à Paris (!) pour leur remettre le fameux papier que l'on sait... Reste le marquis de Faustinières, c'est-à-dire Cabestan lui-même: il n'a apparemment pas beaucoup d'autre mérite que d'être né noble, d'avoir de l'argent et de vouloir le transmettre à sa progéniture...

Arrêtons-nous maintenant pour nous demander quel intérêt un lecteur ou une lectrice de Fraserville (Rivière-duLoup) pouvait bien prendre, en 1892 , à la lecture d'un roman dont les protagonistes et le monde dans lequel ils s'affrontent étaient si étrangers à son propre univers.

Une réponse plausible nous est d'abord suggérée par Ponson du Terrail lui-même qui, partant d'une constatation psychologique toute simple, écrivait: "Les lecteurs n'aiment pas se trouver en face d'acteurs de leur monde. L'homme du peuple n'aime pas l'homme du peuple, ni le marquis le marquis [...] Il faut les malheurs d'une duchesse pour faire pleurer les blanchisseuses ${ }^{10}$. Le marquis de Faustinières et sa fortune, objet de tant d'intrigues et de complots, la mort tragique du beau et jeune baron Victor, la quête du petit "prince" des Indes à Paris en passant par Londres: voilà en effet autant d'éléments exotiques par lesquels le rêve pouvait faire irruption dans la vie tranquille et routinière de l'habitant des campagnes et des villages du Bas-Saint-Laurent à la fin du XIXe siècle. Quant à Olympe, ce génie du mal qui tire les ficelles de l'action mélodramatique dans le roman, comment savoir si l'ambition dont elle est dévorée n'a pas été secrètement partagée par nombre de lecteurs et de lectrices d'ici, condamnés comme elle dès leur naissance à la pauvreté et à la non-existence sociale? Si les héros des feuilletons sont "populaires", c'est parce qu'ils offrent au peuple une revanche mythique, comme le suggère Roger Bellet ${ }^{11}$. À ce sujet, dans Le mythe et l'homme, Roger Caillois distingue ce qu'il appelle "la mythologie des situations et celle des héros". Les situations mythiques proviendraient de la projection de conflits psychologiques qui varient selon les individus et le

10 Ibid., p. 209.

11 Ibid. 
type de société à laquelle ils appartiennent. La plupart du temps, l'individu est inconscient de ces conflits en raison des contraintes que la collectivité fait peser sur ses désirs élémentaires, en l'occurrence la fortune et la reconnaissance sociale. Dieu sait si la société de conservation qui prévalait dans le Bas-Saint-Laurent à l'époque qui nous intéresse a dû peser lourd en ce sens! Et Roger Caillois précise:

... l'individu est dans l'impossibilité de sortir de ces conflits, car il ne pourrait le faire que par un acte condamné par la société et, par conséquent, par lui-même dont la conscience est fortement empreinte et, en quelque sorte, garante des interdictions sociales. Le résultat est qu'il est paralysé devant l'acte tabou et qu'il va en confier l'exécution au héros. 12

Par définition, le héros est justement celui qui viole les prohibitions (comme le fait Olympe dans Les voleurs $d u$ grand monde):

... humain, [le héros] serait coupable et, mythique, il ne cesse pas de l'être: il reste souillé de son acte, et la purification, si elle est nécessaire, n'est jamais complète. Mais à la lumière spéciale du mythe, la grandeur, il apparaît justifié inconditionnellement. Le héros est donc celui qui résout le conflit où l'individu se débat: d'où son droit supérieur non pas tant au crime qu'à la culpabilité, la fonction de cette culpabilité idéale étant de flatter l'individu qui la désire sans pouvoir l'assumer. 13

Voilà dans quel sens on peut se demander si les lecteurs du Bas-Saint-Laurent n'ont pas été à l'époque autant, sinon plus, "flattés» que scandalisés par les audaces d'Olympe. $\mathrm{Ne}$ met-on pas dès lors ici le doigt sur l'une des raisons, peut-être la principale, qui expliquent pourquoi le feuilleton a toujours été suspect aux yeux de tous les pouvoirs?

Si maintenant l'on garde à l'esprit la distinction retenue plus haut entre feuilleton-roman et roman-feuilleton, force est de constater que notre corpus est presque exclusivement composé de feuilletons-romans, c'est-à-dire de romans déjà édités que les journaux débitaient pour les publier en tranches hebdomadaires et le plus souvent, comme le voulait

12 Roger Caillois, Le mythe et l'homme, coll. "Idées ", Paris, Gallimard, 1972, p. 24-25.

13 lbid., p. 25-26. 
la coutume, au rez-de-chaussée de l'une ou l'autre page de leurs publications. Cette pratique semble avoir prévalu dans la plupart des journaux québécois qui, de toute évidence, n'avaient pas comme la presse parisienne les moyens de se payer des feuilletonistes. Il faut cependant mentionner que certains de nos romanciers les plus connus ont d'abord publié en feuilletons des œuvres qui ne parurent ensuite qu'en librairie. Citons entre autres Charles Guérin de P.-J.-O. Chauveau: le roman parut dans L'album litteraire et musical de la Revue canadienne (3 février 1846-mars 1847) pour n'être édité que sept ans plus tard (1853) par John Lovell à Montréal. Laure Conan fit également paraître Angéline de Montbrun en feuilleton dans $L a$ revue canadienne (juin 1888août 1882); le roman ne sortit en librairie qu'en 1884 (Québec, Léger Brousseau). Un dernier exemple: "Maria Chapdelaine parut d'abord [en feuilleton] dans Le temps en France en 1914. sa première édition en livre se fit à Montréal en $1916^{14}$. Dans le Bas-Saint-Laurent ${ }^{15}$, Charles De Guise doit être considéré comme le premier "feuilletoniste". Natif de Kamouraska, ce médecin vint se fixer à Sainte-Anne-de-laPocatière en 1856. Il mourut en 1884. Sa belle légende, $L e$ cap au diable, parut d'abord sous forme de roman-feuilleton dans la Gazette des campagnes (4 novembre 1862-16 février 1863) avant d'être publiée en volume par l'éditeur de ce journal en 1863. L'histoire, qui commence en Acadie au temps de la déportation, se termine à Kamouraska, plus précisément à l'endroit qui porte toujours le nom de Cap-au-Diable. Il s'agit en fait d'un mélodrame riche en péripéties maritimes et qui témoigne d'une maîtrise étonnante des techniques de la narration discontinue.

Le 12 août 1880, Le nouvelliste de Rimouski, sous la rubrique plutôt inattendue "Bibliographie», annonçait: «Un ami de notre feuille veut bien nous communiquer quelques extraits pris au hasard dans un ouvrage qui se prépare pour l'impression, et a pour titre Nouvelles gaspésiennes. Le Livre Premier est intitulé: Moeurs, récits, légendes, lieutenantsgouverneurs de Gaspé [...] Le Livre Second se compose de

14 Bernard Proulx, Le roman du territoire, "Les cahiers du Département d'études littéraires ", Montréal, UQAM, 1987, p. 230.

15 Voir Gilles Lamontagne, "Le feuilleton, mode de diffusion de la littérature bas-laurentienne (1861-1959) ", La revue d'histoire du Bas-Saint-Laurent, vol. X, nos 2-3 (mai-décembre 1984), pp. 100-108. 
80

notices sur les principales paroisses de la Gaspésie..." Suit la publication, sous l'anonymat, de la première tranche du feuilleton. Il y en aura huit (du 12 août au 14 octobre 1880). Sauf erreurs, les Nouvelles gaspésiennes n'ont jamais paru en librairie telles quelles. Les Archives nationales du Québec possèdent toutefois un tapuscrit de cent trente-quatre pages, sans date, ayant pour auteur Monseigneur F.-X. Bossé 16 . ce prêtre appartint vraisemblablement au diocèse de Rimouski puisqu'il dédie son ouvrage " $\mathrm{A}$ Monseigneur Jean Langevin, premier évêque de Saint-Germain de Rimouski, fondateur de ce diocèse [1867], pontife au zèle infatigable, habile administrateur».

La table des matières de Nouvelles gaspésiennes fait état de seize chapitres. En réalité, ceux-ci (p. 8-86) composent uniquement le Livre Premier dont parlait le journal et que l'auteur présente dans sa préface en même temps que le Livre Second (dix autres chapitres, p. 86-134) dont il n'est pas question dans la table des matières.

Enfin, L'Action sociale de Québec publia en 1931 un texte anonyme, Récits d'autrefois. Histoires et légendes du BasSaint-Laurent (117 p.) qui nous ramène cinquante ans plus tôt à l'anonymat et au contenu du feuilleton publié par Le nouvelliste de Rimouski ainsi qu'au tapuscrit de Nouvelles gaspésiennes. Il est en effet évident qu'on est ici en présence de la même "matière ", publiée en trois versions différentes. Voici, à titre d'exemple, le début du $1^{\text {er }}$ chapitre des trois textes.

1. Feuilleton (anonyme, Le nouvelliste de Rimouski, 12 août 1880):

La Noble «morvaille" des balustres. Épisode extraordinaire de la vie d'un curé de l'Islet.

Les "habitants" de la côte de Beaupré appellent leurs jolies paroisses «paradis terrestre ". C'est un fait que tout semble y sourire: tout y respire le bonheur. Les "sorciers" de l'île d'Orléans plaident, eux, pour leurs foyers, et soutiennent, envers et contre tous, que rien n'égale le charme de leurs parages pittoresques...

2. Nouvelles gaspésiennes (Mgr Bossé, tapuscrit des Archives nationales du Québec, sans date): 
Beaupréens. Sorciers. Mangeurs de crêpes. Calumets. La palme est au rang des Belles-Amours.

- Hourra pour CHEZ NOUS, la terre du blé, des gras paturage [sic], des riches vergers, le paradis terrestre pour certain.

C'est là votre cri du cceur habitants de la côte de Beaupré... 1931):

3. Récits d'autrefois... (anonyme, L'Action sociale, Québec,

Beaupréens. Sorciers. Mangeurs de crêpes. Calumets. La palme est au rang des Belles-Amours.

- Hourra pour chez nous, la terre du blé, des gras pâturages, des riches vergers, le paradis terrestre, pour certain. C'est là votre cri du cœur, habitants de la côte de Beaupré...

Comme on peut s'en rendre compte, le texte le plus démarqué est celui du feuilleton. Correspond-il vraiment à l'envoi de l'auteur (anonyme), comme l'affirme la présentation du journal ou s'agit-il plutôt d'un repiquage effectué par le journal à même une copie du tapuscrit de Mgr Bossé ? Malgré ce que suggèrent les trop brefs extraits reproduits ici, il existe entre les deux dernières versions des différences parfois notables, surtout dans les derniers chapitres.

Quoi qu'il en soit, les trois textes s'articulent autour d'une sorte de tronc commun dont voici les éléments de base: on est vers 1850; le narrateur est originaire du rang des BellesAmours dans la paroisse de l'Islet. À la fois témoin et acteur, il raconte d'abord des histoires de tables tournantes et d'ensorcellements. Pour changer d'air, il décide un jour de s'embarquer sur une barge en partance pour Percé. Les arrêts qu'il effectuera au cours du voyage serviront occasionnellement de prétexte à raconter des légendes comme celle du Cap-Bonamy, des histoires de revenants, de maisons hantées et certains tours que les "Percéens " prenaient un malin plaisir à jouer aux "Gasponiens", c'est-à-dire aux habitants du village de Gaspé.

La troisième version, Récits d'autrefois..., a déjà été attribuée à tort à Damase Potvin. Aujourd'hui, on croit plutôt qu'elle est l'œuvre d'un certain abbé François Gagné, du diocèse de Québec. Ce dernier, après avoir modifié le titre, a remanié le texte de Mgr Bossé qui comprend désormais vingt-deux chapitres répartis en trois livres. Empruntant ensuite aux romanciers du XVIII siècle un artifice littéraire bien connu, l'abbé Gagné compose une introduction dans laquelle un visiteur de passage 
82

trouve dans la bibliothèque d'un curé de campagne un cahier intitulé: Gaspésie; histoires et légendes. Ce sont ces récits qu'avec l'autorisation du prêtre le visiteur va publier. Cela dit, on constate que l'abbé Gagné a délesté le texte de Mgr Bossé de la plupart de ses prétentions historiques. Enfin, le style de l'ecclésiastique de Québec est beaucoup plus alerte et teinté d'un humour moins appuyé que celui de Mgr Bossé.

\section{***}

Sans contredit, de tous les textes de notre corpus, le plus étonnant roman-feuilleton demeure celui qu'on peut lire dans Le progrès $d u$ golfe de Rimouski (21 avril-7 juillet 1911) sous le titre Le juif, l'éternel juif. Il s'agit d'une commande du journal à l'un de ses collaborateurs (un ecclésiastique?) dont le nom, Anastase Deschamps, est presque certainement un pseudonyme. Cette "nouvelle", comme l'appelle abusivement le journal, n'a jamais été publiée ailleurs.

Tout commence avec la parution dans la feuille rimouskoise d'une série de cinq billets (10 mars-7 avril 1911) sous la signature de Julius. Celui-ci y dénonce un colporteur juif qui vend des "capots" de chat d'une qualité plus que douteuse dans la soi-disant région de Safabihen de Mirouski (SaintFabien de Rimouski, évidemment)... en Pologne ${ }^{17}$ !

Quelques jours à peine après la parution du premier billet, intitulé «Juif et capot de chat», un marchand de Rimouski, Salomon Waiser, à tort ou à raison, se sentit visé. Aussi fit-il parvenir au journal une longue déclaration notariée (17 mars 1911) dans laquelle il affirmait, non sans courage et avec beaucoup de dignité:

Je suis né de race juive, purement juive, venant de Roumanie [...] Je suis depuis quatre ans à Rimouski où j'exerce un commerce général [...] Je resterai à Rimouski aussi longtemps que je serai vivant. Aucune menace, aucune injure venant d'aucune personne [...] en un mot rien ni personne ne m'obligera à partir de Rimouski quels que soient les procédés que l'on emploiera à mon égard [...] Je suis capable de dire le nombre de personnes avec qui j'ai fait affaire, même de les

17 On songe à Alfred Jarry situant l'épopée burlesque d'Ubu en Pologne, c'esta-dire Nulle Part. 
nommer - il n'y en a pas moins de trois mille — et qui ont toujours été parfaitement bien servies [...] Je suis d'aussi bonne race que qui que ce soit [...] Le peuple auquel j'appartiens n'est pas plus criminel, ni plus lâche, ni plus immoral, ni plus voleur, ni plus menteur que n'importe lequel autre peuple de l'univers [...]

[signé] Salomon Waiser. En présence de [signé] J.-Eudore Couture, notaire public.

Julius ne se laissa pas ébranler par la protestation du marchand. Tout en se défendant avec beaucoup de perfidie de n'avoir jamais attaqué « ni Waiser, ni les Juifs, ni en gros, ni en détail", il revint à la charge en citant sa victime pour s’indigner aussitôt après: "Quoi! ma race française et chrétienne ne vaut pas mieux que la race juive et infidèle qui a traîné sur tous les boulevards et dans les carrefours de l'ancien et du nouveau monde?" (31 mars) On voit le ton férocement antisémite et raciste des billets publiés avec la complicité du directeur du journal qui applaudissait en comptant les coups: «Un mot du directeur? Le voici en trois mots: continuez, pardi, continuez!» (ibid.)

On continua, en effet. Du simple billet, on passa au feuilleton dont la première tranche est coiffée du titre alambiqué: "Le juif, l'éternel juif. Un autre canadien et un autre peutêtre... le même capot de chat. Histoire vécue. Nouvelle inédite pour Le progrès du golfe par Anastase Deschamps ». Il y a fort à parier que derrière ce pseudonyme se cache le chroniqueur qui signait Julius... Le style et le ton des billets sont en tout cas tout à fait identiques à ceux de la soi-disant nouvelle.

À certains égards, ce feuilleton relativement long, compte tenu de la minceur du sujet (n'oublions pas qu'il s'étire sur douze semaines), n'est pas sans rappeler ceux qui proliférèrent en France sous le Second Empire (1850-1870), époque de dégénérescence du genre à laquelle appartient Ponson du Terrail. (L'âge d'or du feuilleton, celui du feuilleton "classique", si l'on peut dire, avec Frédéric Soulié, Paul Féval et Eugène Sue, se situe sous le règne de Louis-Philippe (1830-1848). Donc, Le juif, l'éternel juif s'apparente un peu au feuilleton de la deuxième période, celle de l'abâtardissement du roman populaire. Mêmes extravagances, mêmes procédés cousus de fil blanc pour tenter de remettre sur ses rails une intrigue qui s'en va dans tous les azimuts, mêmes empiètements du discours de l'auteur sur le récit du narrateur qui se traduisent 
84

ici par des élans de chauvinisme, contredits par de longues remontrances à l'adresse de l'un des protagonistes, JeanBaptiste Lachance, personnification symbolique évidente du Canadien français bonasse, naïf, toujours prêt à céder à toutes les tentations du modernisme. De plus, le texte de Deschamps ne manque pas d'humour, mais d'un humour qu'on ne peut malheureusement savourer sans mauvaise conscience, étant donné le racisme incroyable qu'il distille.

Total des feuilletons recensés: 144

(147 si l'on tient compte du fait que trois d'entre eux ont été publiés deux fois)

Tableau I

\begin{tabular}{|l|l|}
\hline $1861-1899$ (39 ans) & $1900-1950$ (50 ans) \\
\hline $\begin{array}{l}78 \text { feuilletons } \\
\text { (44 anonymes) }\end{array}$ & $\begin{array}{l}69 \text { feuilletons } \\
(19 \text { anonymes })\end{array}$ \\
\cline { 2 - 2 } & $\begin{array}{l}1918-1950 \\
38 \text { feuilletons } \\
(6 \text { anonymes, dont } \\
\text { les } 3 \text { ciné-romans })\end{array}$ \\
\hline
\end{tabular}

Tableau II

\begin{tabular}{|l|l|}
\hline $1861-1899$ & $1900-1950$ \\
\hline $\begin{array}{l}78 \text { feuilletons } \\
\text { (13 écrits par des } \\
\text { Canadiens }\end{array}$ & $\begin{array}{l}\text { 69 feuilletons } \\
\text { (31 écrits par des } \\
\text { Canadiens) }\end{array}$ \\
\cline { 2 - 2 } & $\begin{array}{l}1920-1950 \\
34 \text { feuilletons } \\
\text { (25 écrits par des } \\
\text { Canadiens) }\end{array}$ \\
\hline
\end{tabular}


Soixante des cent quarante-quatre feuilletons qui composent notre corpus ont paru anonymement. Dix-neuf de ces auteurs anonymes ont pu être identifiés avec certitude; pour onze autres, un doute subsiste. Les catalogues consultés indiquent en effet que certains romans, portant des titres identiques, doivent être attribués à des auteurs différents. Ainsi, par exemple, il existe au moins cinq L'orpheline, trois Mercedes et deux Pauvre Jacques... Seule la confrontation des feuilletons avec les textes publiés en librairie pourrait permettre d'identifier les auteurs.

L'attribution de leur nationalité aux auteurs soulève aussi des problèmes reliés le plus souvent, par définition, à celui de l'anonymat; elle est donc sujette à caution. En effet, s'appuyer seulement sur le facteur référentiel d'un feuilleton pour déduire que son auteur est de telle ou telle nationalité ne met pas à l'abri de l'erreur. Cela dit et, par conséquent, sous toutes réserves, quelque cent trois de nos cent quarantequatre feuilletons auraient des auteurs non canadiens, plus précisément des Français, dans la plupart des cas; les quarante et un autres auraient été écrits par des Canadiens et, de ce nombre, dix-huit ont été signés par des écrivains originaires du Bas-Saint-Laurent.

\section{Liste des journaux porteurs de feuilletons, par ordre d'ancienneté}

1. La gazette des campagnes

Saint-Louis-de-Kamouraska, septembre-novembre 1861 ; Sainte-Anne-de-la-Pocatière, 21 septembre 1861-mars 1895. Bimensuel, septembre 1861-octobre 1867; hebdomadaire, avril 1868-1895. D'abord imprimé à Québec, chez Darveau; à partir du numéro 7 (3 avril 1862), imprimé à La Pocatière.

Nombre de feuilletons publiés: 14 .

2. La voix du golfe

Rimouski, 25 juin 1867-18 juillet 1871. Hebdomadaire.

Nombre de feuilletons publiés: 13 .

3. Le courrier de Rimouski

Rimouski, 2 septembre 1871-21 novembre 1873, Bihebdomadaire. 
86

Nombre de feuilletons publiés: 6 .

4. Le nouvelliste de Rimouski

Rimouski, 23 décembre 1876-1881. Les huit premiers numéros publient deux pages en anglais sous le titre The Rimouski Star. Hebdomadaire.

Nombre de feuilletons publiés: 8 .

5. L'écho de Fraserville

Fraser ville (Rivière-du-Loup), 31 mai 1884. Hebdomadaire.

Nombre de feuilletons publiés: 1 .

6. Le jour

Fraserville (Rivière-du-Loup), 9 décembre 1881-23 mars 1888. Imprimé à Lévis, mais daté de Fraserville. Hebdomadaire.

Nombre de feuilletons publiés: 12.

7. Le journal de Fraserville

Fraserville (Rivière-du-Loup), 26 décembre 1888 1927). Hebdomadaire. Ce journal, dont le propriétaire était le libraire-imprimeur de Lévis J.-E. Mercier, est le même que Le jour qui, après s'être appelé Le progrès de Fraserville, est devenu Le journal de Fraser. ville, nom qu'il conservera jusqu'à sa disparition.

Nombre de feuilletons publiés: 5 .

8. L'écho du golfe

Rimouski, 2 juin 1885-18 novembre 1886. Hebdomadaire.

Nombre de feuilletons publiés: 3 .

9. Le courrier de Fraserville

Fraserville (Rivière-du-Loup), 16 novembre 1887-20 décembre 1889. Hebdomadaire.

Nombre de feuilletons publiés: 7 .

10.Le Saint-Laurent

Rivière-du-Loup, 19 novembre 1895-30 juin 1876 . Bihebdomadaire, 1895-1899; hebdomadaire, 1899... 
Nombre de feuilletons publiés: 50 .

11.Bulletin politique

Rivière-du-Loup, 24 février 1899-juin 1900.

Nombre de feuilletons publiés: 2 .

12.Le progrès du golfe

Rimouski, 15 avril 1904-6mai 1970. Hebdomadaire

Nombre de feuilletons publiés: 14 .

\section{L'echo du Bas-Saint-Laurent}

Rimouski, 3 mars 1933-29 avril 1970. Hebdomadaire.

Nombre de feuilletons publiés: 14 . 